\title{
LIFTING UNCONDITIONALLY CONVERGING SERIES AND SEMIGROUPS OF OPERATORS
}

\author{
Manuel González and Antonio Martínez-Abejón
}

\begin{abstract}
We introduce and study two semigroups of operators $\mathcal{U}_{+}$and $\mathcal{U}_{-}$, defined in terms of unconditionally converging series. We prove a lifting result for unconditionally converging series that allows us to show examples of operators in $\mathcal{U}_{+}$. We obtain perturbative characterisations for these semigroups and, as a consequence, we derive characterisations for some classes of Banach spaces in terms of the semigroups. If $\mathcal{U}_{+}(X, Y)$ is non-empty and every copy of $c_{0}$ in $Y$ is complemented, then the same is true in $X$. We solve the perturbation class problem for the semigroup $\mathcal{U}_{-}$, and we show that a Banach space $X$ contains no copies of $\ell_{\infty}$ if and only if for every equivalent norm $|\cdot|$ on $X$, the semiembeddings of $(X,|\cdot|)$ belong to $\mathcal{U}_{+}$.
\end{abstract}

\section{INTRODUCTION}

Tauberian operators, introduced by Kalton and Wilansky [12], are useful in Banach space theory because they preserve some isomorphic properties of sets in Banach spaces. For example, the second factor in the factorisation given in [5] is tauberian and, since tauberian operators preserve the relative weak compactness of bounded sets, it follows that weakly compact operators factorise through reflexive Banach spaces. We refer to the survey [7] for further information about tauberian operators.

The class of tauberian operators is a semigroup and has analogous properties to that of upper semi-Fredholm operators, replacing finite dimensional spaces by reflexive spaces. We refer to $[\mathbf{7}, \mathbf{1 0}]$ for details. Moreover, upper semi-Fredholm operators, tauberian operators and other semigroups of operators defined in terms of sequences admit perturbative characterisations [10].

Here we define two new semigroups of operators, denoted $\mathcal{U}_{+}$and $\mathcal{U}_{-}$, in terms of the action of the operators over unconditionally converging series. We obtain a perturbative characterisation: An operator $T \in \mathcal{B}(X, Y)$ belongs to $\mathcal{U}_{+}$if and only if for every compact operator $K \in \mathcal{B}(X, Y)$ the kernel $N(T+K)$ contains no copies of $c_{0}$. As a consequence we derive an algebraic characterisation of operators in $\mathcal{U}_{+}$, we show that a Banach space $X$ contains no copies of $\ell_{\infty}$ if and only if every semiembedding of $X$ belongs to $\mathcal{U}_{+}$, and

Received 13th May, 1997

Supported in part by DGICYT Grant PB 94-1052 (Spain).

Copyright Clearance Centre, Inc. Serial-fee code: 0004-9729/98 \$A2.00+0.00. 
we characterise Banach spaces whose non-reflexive (respectively, infinite dimensional) subspaces contain $c_{0}$ in terms of $\mathcal{U}_{+}$.

We prove a lifting result for unconditionally converging series, analogous to Lohman's lifting for weakly Cauchy sequences [15]. As a consequence, we show that operators with closed range and kernel containing no copies of $c_{0}$ belong to $\mathcal{U}_{+}$, and operators with closed range and cokernel containing no complemented copies of $\ell_{1}$ belong to $\mathcal{U}_{-}$. We refer to [9] for other lifting results for sequences.

We also prove that operators in $\mathcal{U}_{+}$preserve an isomorphic property: if there exists an operator $T \in \mathcal{U}_{+}(X, Y)$ and every subspace of $Y$ isomorphic to $c_{0}$ is complemented, then the same is true for $X$. Separable spaces, or more generally, weakly compactly generated spaces, satisfy this property.

For the dual semigroup $\mathcal{U}_{-}$we also obtain a perturbative characterisation: $T \in$ $\mathcal{B}(X, Y)$ belongs to $\mathcal{U}_{-}$if and only if for every compact operator $K \in \mathcal{B}(X, Y)$ the cokernel $Y / \overline{R(T)}$ contains no complemented copies of $\ell_{1}$. As a consequence, we derive characterisations of Banach spaces whose non-reflexive (respectively, infinite dimensional) quotients contain a complemented copy of $\ell_{1}$ in terms of $\mathcal{U}_{-}$. We also solve the perturbation class problem for the semigroup $\mathcal{U}_{-}$: given an operator $K \in \mathcal{B}(X, Y)$, we have $T+K \in \mathcal{U}_{-}$for every $T \in \mathcal{U}_{-}(X, Y)$ if and only if the conjugate $K^{*}$ is unconditionally converging.

We use standard notations: $X$ and $Y$ are Banach spaces and $B_{X}$ denotes the closed unit ball of $X$. The class of (bounded linear) operators from $X$ to $Y$ is $\mathcal{B}(X, Y)$, the dual of $X$ is $X^{*}$, and given an operator $T \in \mathcal{B}(X, Y)$, we denote by $T^{*}: Y^{*} \longrightarrow X^{*}$ the conjugate operator of $T$, by $R(T)$ and $N(T)$ the range and kernel of $T$, and by $Y / \overline{R(T)}$ the cokernel of $T$. Moreover, $\mathbb{N}$ is the set of all positive integers. We identify $X$ with a subspace of $X^{* *}$.

\section{The Semigroups}

Recall that a series $\sum_{n=1}^{\infty} x_{n}$ in a Banach space $X$ is weakly unconditionally Cauchy if $\sum_{n=1}^{\infty}\left|x^{*}\left(x_{n}\right)\right|<\infty$ for all $x^{*} \in X^{*}$. A series is unconditionally converging if every subseries is convergent.

An operator $T \in \mathcal{B}(X, Y)$ is said to be unconditionally converging, denoted $T \in$ $\mathcal{U}(X, Y)$, if it takes weakly unconditionally Cauchy series into unconditionally converging series. The following characterisation will be useful. We refer to [18, p.270] for a proof.

PROPOSITION 2.1. An operator $T \in \mathcal{B}(X, Y)$ is unconditionally converging if and only if given a subspace $M$ of $X$, if the restriction $\left.T\right|_{M}$ is an isomorphism then $M$ contains no copies of $c_{0}$.

The definition of the semigroup $\mathcal{U}_{+}$is opposite in some sense to that of the uncon- 
ditionally converging operators.

Definition 2.2: An operator $T \in \mathcal{B}(X, Y)$ belongs to the class $\mathcal{U}_{+}$if for every weakly unconditionally Cauchy series $\sum_{n=1}^{\infty} x_{n}$ in $X$, if $\sum_{n=1}^{\infty} T x_{n}$ is unconditionally converging then $\sum_{n=1}^{\infty} x_{n}$ is unconditionally converging

REMARK 2.3. (a) It readily follows from the definition that the class $\mathcal{U}_{+}$is stable under products and under unconditionally converging perturbations:

$$
\begin{aligned}
& T \in \mathcal{U}_{+}(X, Y) \text { and } S \in \mathcal{U}_{+}(Y, Z) \Rightarrow S T \in \mathcal{U}_{+}(X, Z) ; \\
& T \in \mathcal{U}_{+}(X, Y) \text { and } K \in \mathcal{U}(X, Y) \Rightarrow T+K \in \mathcal{U}_{+}(X, Y) .
\end{aligned}
$$

(b) Since a Banach space $X$ contains no copies of $c_{0}$ if and only if every weakly unconditionally Cauchy series in $X$ is unconditionally converging [2, Theorem 5], we have

$$
\dot{T} \in \mathcal{U}_{+}(X, Y) \Rightarrow N(T) \text { contains no copies of } c_{0} .
$$

Now we prove a lifting result for unconditionally converging series and derive some consequences. Given a subspace $M$ of a Banach space $X$, we denote by $q_{M}: X \rightarrow X / M$ the quotient map.

THEOREM 2.4. Let $M$ be a subspace of $X$ containing no copies of $c_{0}$. If $\sum_{n=1}^{\infty} x_{n}$ is a weakly unconditionally Cauchy series in $X$, and $\sum_{n=1}^{\infty} q_{M} x_{n}$ is unconditionally converging, then $\sum_{n=1}^{\infty} x_{n}$ is unconditionally converging

ProOF: Clearly, it is enough to show that $\sum_{n=1}^{\infty} x_{n}$ is convergent.

Suppose that $\sum_{n=1}^{\infty} x_{n}$ is non-convergent. Then there are a number $\delta>0$ and integers $1 \leqslant m_{1} \leqslant n_{1}<m_{2} \leqslant n_{2}<\ldots$ so that, denoting $y_{k}:=x_{m_{k}}+\ldots+x_{n_{k}}$, we have $\left\|y_{k}\right\|>\delta$.

The sequence $\left(y_{k}\right)$ is weakly null and bounded away from 0 . Therefore, using the Bessaga-Pelczynski selection principle $[2, \mathrm{C} .1]$, we can select a basic subsequence $\left(z_{n}\right)$ of $\left(y_{k}\right)$. This subsequence is equivalent to the unit vector basis of $c_{0}$ because $\sum_{n=1}^{\infty} z_{n}$ is weakly unconditionally Cauchy [2, Lemma 1$]$.

Moreover, since $\sum_{n=1}^{\infty} q_{M} x_{n}$ is unconditionally converging, we have that $\left(q_{M} z_{n}\right)$ converges in norm to 0 . Then we can find a sequence $\left(w_{n}\right)$ in $M$ such that $\lim _{n \rightarrow \infty}\left\|w_{n}-z_{n}\right\|=0$, and a standard perturbation argument for basic sequences implies that a subsequence of $\left(w_{n}\right)$ is equivalent to the basis of $c_{0}$, which gives a contradiction. 
CoRollary 2.5. If $T \in \mathcal{B}(X, Y)$ has closed range and its kernel contains no copies of $c_{0}$, then $T \in \mathcal{U}_{+}$.

Proof: It is enough to observe that $T$ can be written as the composition of the quotient map $X \longrightarrow X / N(T)$ and an isomorphism (into).

The following result is well-known. We prove it as an application of our lifting result.

COROLlary 2.6. The class of Banach spaces that contain no copies of $c_{0}$ has the three-space property.

Proof: Let $M$ be a subspace of $X$ and assume $M$ and $X / M$ contain no copies of $c_{0}$. If we denote by $Q: X \longrightarrow X / M$ the quotient map, given a weakly unconditionally Cauchy series $\sum_{n=1}^{\infty} x_{n}$, we have that $\sum_{n=1}^{\infty} Q x_{n}$ is weakly unconditionally Cauchy in $X / M$; hence unconditionally converging because $X / M$ contains no copies of $c_{0}$, and applying the lifting result we conclude that $\sum_{n=1}^{\infty} x_{n}$ is unconditionally converging.

The operators in $\mathcal{U}_{+}$can be characterised by their action over sequences equivalent to the unit vector basis of $c_{0}$ and in terms of the kernels of the perturbations by operators in $\mathcal{U}$.

Proposition 2.7. For $T \in \mathcal{B}(X, Y)$ the following statements are equivalent:

(a) $T \in \mathcal{U}_{+}(X, Y)$;

(b) if $\left(x_{n}\right) \subset X$ is equivalent to the unit vector basis of $c_{0}$ then there exists $k \in \mathbb{N}$ such that $\left(T x_{n}\right)_{n>k}$ is equivalent to the unit vector basis of $c_{0}$;

(c) there is no (normalised) sequence $\left(x_{n}\right)$ in $X$ equivalent to the unit vector basis of $c_{0}$ and such that $\lim _{k \rightarrow \infty} T x_{k}=0$.

Proof: $(a) \Rightarrow(b)$ Assume $\left(x_{n}\right)_{n}$ is a sequence in $X$ equivalent to the unit vector basis of $c_{0}$, but $\left(T x_{n}\right)_{n>k}$ is equivalent to this basis for no $k \in \mathbb{N}$. Then we can find a sequence of scalars $\left(a_{n}\right)$ such that $\left|a_{n}\right| \leqslant 1$ for all $n$ and a sequence of integers $1 \leqslant m_{1} \leqslant$ $n_{1}<m_{2} \leqslant n_{2}<\cdots$ so that, denoting $y_{k}:=a_{m_{k}} x_{m_{k}}+\ldots+a_{n_{k}} x_{n_{k}}$, we have that $\left(y_{k}\right)$ is equivalent to the unit vector basis of $c_{0}$, but $\left\|T y_{k}\right\| \rightarrow 0$. By passing to a subsequence we may assume $\left\|T y_{k}\right\|<2^{-k}$. Then $\sum_{k=1}^{\infty} T y_{k}$ is unconditionally converging, and we conclude that $T \notin \mathcal{U}_{+}$.

(b) $\Rightarrow(c)$ is trivial.

(c) $\Rightarrow$ (a) Assume that $T \notin \mathcal{U}_{+}(X, Y)$. Then there is a weakly unconditionally Cauchy series $\sum_{k=1}^{\infty} z_{k}$ in $X$ which is not unconditionally converging, such that $\sum_{k=1}^{\infty} T z_{n}$ is unconditionally converging. Now, proceeding as in the proof of Theorem 2.4 , we obtain a sequence $\left(y_{k}\right)$ equivalent to the unit vector basis of $c_{0}$ and such that $\lim _{k \rightarrow \infty} T y_{k}=0$, and the normalised sequence given by $x_{k}:=\left\|y_{k}\right\|^{-1} y_{k}$ shows that (c) fails. 
THEOREM 2.8. An operator $T \in \mathcal{B}(X, Y)$ belongs to $\mathcal{U}_{+}$if and only if for every compact operator $K \in \mathcal{B}(X, Y)$ the kernel $N(T+K)$ contains no copies of $c_{0}$.

Proof: The direct implication was shown in Remark 2.3 .

For the converse, assume $T$ does not belong to $\mathcal{U}_{+}$. By Proposition 2.7, we can find $\left(x_{n}\right)$ in $X$ equivalent to the unit vector basis of $c_{0}$, and $\left(f_{n}\right)$ bounded in $X^{*}$ such that $f_{i}\left(x_{j}\right)=\delta_{i j}$ and $\left\|f_{n}\right\|\left\|T x_{n}\right\|<2^{n}$. Then

$$
K x:=-\sum_{n=1}^{\infty} f_{n}(x) T x_{n}
$$

defines a compact operator $K \in \mathcal{B}(X, Y)$ such that $N(T+K)$ contains the subspace generated by $\left(x_{n}\right)$.

As a consequence of the perturbative characterisation, we derive "algebraic" characterisations.

COROLlaRY 2.9. For $T \in \mathcal{B}(X, Y)$, the following statements are equivalent:

(a) $T \in \mathcal{U}_{+}(X, Y)$;

(b) for every Banach space $Z$ and every $L \in \mathcal{B}(Z, X)$, we have $T L \in \mathcal{U}(Z, Y)$ only if $L \in \mathcal{U}(Z, X)$;

(c) for every subspace $M \subset X$, if the restriction $\left.T\right|_{M}$ belongs to $\mathcal{U}(M, Y)$, then $M$ contains no copies of $c_{0}$.

Proof: (a) $\Rightarrow$ (b) Assume $T \in \mathcal{U}_{+}(X, Y)$ and let $L \in \mathcal{B}(Z, X)$ be an operator such that $T L \in \mathcal{U}(Z, Y)$. Let $\sum z_{k}$ be a weakly unconditionally Cauchy series in $Z$. Thus $\sum T L z_{k}$ is unconditionally converging, and since $T \in \mathcal{U}_{+}(X, Y)$, the series $\sum L z_{k}$ must be unconditionally converging.

(b) $\Rightarrow$ (c) It is enough to observe that the inclusion operator $i_{M}: M \longrightarrow X$ belongs to $\mathcal{U}(M, X)$ if and only if $M$ does not contain copies of $c_{0}$ [2, Theorem 5].

(c) $\Rightarrow$ (a) Assume $T \notin \mathcal{U}_{+}(X, Y)$. By Theorem 2.8 there is a compact operator $K \epsilon$ $\mathcal{B}(X, Y)$ such that $M:=N(T+K)$ contains a copy of $c_{0}$. As $T i_{M}=-K i_{M}$, we have that $T i_{M}$ is compact, hence $T i_{M} \in \mathcal{U}$, and $M$ contains a copy of $c_{0}$.

Recall that $T \in \mathcal{B}(X, Y)$ is said to be upper semi-Fredholm if it has closed range and finite dimensional kernel, and $T$ is said to be tauberian if its second conjugate $T^{* *} \in$ $\mathcal{B}\left(X^{* *}, Y^{* *}\right)$ satisfies $T^{* *}\left(X^{* *} \backslash X\right) \subset Y^{* *} \backslash Y$. We denote by $\mathcal{F}_{+}(X, Y)$ and $\mathcal{T}_{+}(X, Y)$ the classes of upper semi-Fredhom operators and tauberian operators, respectively. Both classes admit a perturbative characterisation. The result for $\mathcal{F}_{+}$is classic.

Proposition 2.10. [10, Theorem 1.a] An operator $T \in \mathcal{B}(X, Y)$ belongs to $\mathcal{F}_{+}$(respectively, $\mathcal{T}_{+}$) if and only if for every compact operator $K \in \mathcal{B}(X, Y)$ the kernel $N(T+K)$ is finite dimensional (respectively, reflexive).

COROLlary 2.11. For every pair of Banach spaces $X, Y$ we have

$$
\mathcal{F}_{+}(X, Y) \subset \mathcal{T}_{+}(X, Y) \subset \mathcal{U}_{+}(X, Y)
$$


REMARK 2.12. Although the components of the semigroup $\mathcal{F}_{+}$are open subsets of $\mathcal{B}(X, Y)$, this is not always true for the components of $\mathcal{T}_{+}$and $\mathcal{U}_{+}$. This fact can be seen using an example similar to one given in [1].

In the space $\ell_{2}\left(c_{0}\right):=\left\{\left(x_{n}\right): x_{n} \in c_{0}\right.$ and $\left.\sum_{n=1}^{\infty}\left\|x_{n}\right\|^{2}<\infty\right\}$, we consider the operator $T \in \mathcal{B}\left(\ell_{2}\left(c_{0}\right), \ell_{2}\left(c_{0}\right)\right)$ given by $T\left(x_{n}\right):=\left(x_{n} / n\right)$. It easily follows from the definition that $T$ is tauberian, hence it belongs to $\mathcal{U}_{+}$. However, the operators $\mathcal{T}_{n} \in$ $\mathcal{B}\left(\ell_{2}\left(c_{0}\right), \ell_{2}\left(c_{0}\right)\right)$ given by

$$
T_{n}\left(x_{1}, x_{2}, \ldots\right):=\left(x_{1}, \ldots, x_{n}, 0,0, \ldots\right)
$$

satisfy $\lim _{n}\left\|T-T_{n}\right\|=0$ and $\operatorname{ker}\left(T_{n}\right)$ contains a copy of $c_{0}$ for every $n$. Hence $T$ belongs to the boundaries of $\mathcal{U}_{+}\left(\ell_{2}\left(c_{0}\right), \ell_{2}\left(c_{0}\right)\right)$ and $\mathcal{T}_{+}\left(\ell_{2}\left(c_{0}\right), \ell_{2}\left(c_{0}\right)\right)$.

As a consequence of the perturbative characterisations, we characterise some classes of Banach spaces. Recall that a Banach space $X$ is said to be hereditarily $c_{0}$ if every infinite dimensional subspace of $X$ contains copies of $c_{0}$.

Proposition 2.13. Let $X$ be a Banach space.

(a) The space $X$ is hereditarily $c_{0}$ if and only if for every $Y$ we have $\mathcal{U}_{+}(X, Y)=$ $\mathcal{F}_{+}(X, Y)$.

(b) Non-reflexive subspaces of $X$ contain copies of $c_{0}$ if and only if for every $Y$ we have $\mathcal{U}_{+}(X, Y)=\mathcal{T}_{+}(X, Y)$.

(c) Reflexive subspaces of $X$ are finite dimensional if and only if for every $Y$ we have $\mathcal{T}_{+}(X, Y)=\mathcal{F}_{+}(X, Y)$.

Proof: (a) If $X$ is not hereditarily $c_{0}$, then it contains an infinite dimensional subspace $M$ containing no copies of $c_{0}$. By Corollary 2.5, the quotient map $q_{M}$ belongs to $U_{+} \backslash \mathcal{F}_{+}$. The direct implication follows directly from Theorem 2.8 .

The proof of the other parts is analogous.

We consider now Banach spaces $X$ such that all their subspaces isomorphic to $c_{0}$ are complemented. This is the case when $X$ is separable, weakly compactly generated, or more generally, when $X$ is weakly compactly determined [6, Lemma VI.2.4]. Next we show that operators in $\mathcal{U}_{+}$preserve this class.

Proposition 2.14. Assume that $\mathcal{U}_{+}(X, Y)$ is non-empty. If every subspace of $Y$ isomorphic to $c_{0}$ is complemented, then the same is true for $X$.

Proof: Let $M$ be a subspace of $X$ isomorphic to $c_{0}$. Taking $T \in U_{+}(X, Y)$, by Proposition 2.7 there is a subspace $N$ of $M$ such that $M / N$ is finite dimensional and the restriction $\left.T\right|_{N}$ is an isomorphism. Since $N$ is isomorphic to $c_{0}$, we have that $T(N)$ is complemented. Now, if $L$ is a complement of $T(N)$, then $T^{-1}(L)$ is a complement of $N$; hence $N$ and $M$ are complemented subspaces of $X$. 
Given a semigroup $\mathcal{S}$ of operators, Lebow and Schechter [13] define the perturbation class $\mathcal{P S}$, for spaces $X, Y$ such that $\mathcal{S}(X, Y)$ is not empty, as follows:

$$
\mathcal{P S}(X, Y):=\{A \in \mathcal{B}(X, Y): T+A \in \mathcal{S} \text { for every } T \in \mathcal{S}(X, Y)\} .
$$

Recall that an operator $T \in \mathcal{B}(X, Y)$ is said to be strictly singular if there is no infinite dimensional $M$ of $X$ such that the restriction $\left.T\right|_{M}$ is an isomorphism. The perturbation class for the upper semi-Fredholm operators contains the strictly singular operators, and it is a well-known open problem whether these classes coincide, even in the case $X=Y[\mathbf{1 7}]$.

We have seen in Remark 2.3 that the perturbation class for $\mathcal{U}_{+}(X, Y)$ contains $\mathcal{U}(X, Y)$. Next we show that they coincide whenever subspaces of $X$ and $Y$ isomorphic to $c_{0}$ are complemented.

Proposition 2.15. Assume that $\mathcal{U}_{+}(X, Y)$ is nonempty and that every subspace of $Y$ isomorphic to $c_{0}$ is complemented. Then we have $\mathcal{P U} \mathcal{U}_{+}(X, Y)=\mathcal{U}(X, Y)$.

Proof: Take $A \in \mathcal{B}(X, Y)$ which is not in $\mathcal{U}$. It follows from Proposition 2.1 that there exists a subspace $M$ of $X$ isomorphic to $c_{0}$ such that the restriction $\left.A\right|_{M}$ is an isomorphism. From the hypothesis and Proposition 2.14, by passing to a complemented subspace of $M$ we may assume that

$$
X=U \oplus M \text { and } Y=V \oplus A(M)
$$

with $U$ and $V$ isomorphic to $X$ and $Y$, respectively.

Now we can define an operator $T \in \mathcal{B}(X, Y)$ such that $\left.T\right|_{M}=\left.A\right|_{M}, T(U) \subset V$ and $\left.T\right|_{U}$ belongs to $\mathcal{U}_{+}$. Clearly $T \in \mathcal{U}_{+}$. However, $T+A \notin \mathcal{U}_{+}$.

Now we introduce the dual semigroup.

DEFINITION 2.16: An operator $T \in \mathcal{B}(X, Y)$ belongs to the class $\mathcal{U}_{-}$if its conjugate operator $T^{*}$ belongs to $\mathcal{U}_{+}$.

We denote by $\mathcal{U}^{d}$ the dual operator ideal of $\mathcal{U}$; that is,

$$
\mathcal{U}^{d}(X, Y):=\left\{T \in \mathcal{B}(X, Y): T^{*} \in \mathcal{U}\right\}
$$

The following characterisation of the operators in $\mathcal{U}^{d}$ will be useful. We refer to [18, Lemma p.272] for a proof.

Proposition 2.17. An operator $T \in \mathcal{B}(X, Y)$ belongs to $\mathcal{U}^{d}$ if and only if there is no subspace $M$ of $X$ isomorphic to $\ell_{1}$ such that the restriction $\left.T\right|_{M}$ is an isomorphism and $T(M)$ is complemented in $Y$.

REMARK 2.18. (a) It is not difficult to derive from the previous result that the dual $X^{*}$ of a Banach space $X$ contains no copies of $c_{0}$ if and only if $X$ contains no complemented copies of $\ell_{1}[2$, Theorem 4]. 
(b) Similarly as in the case of $\mathcal{U}_{+}$, the class $\mathcal{U}_{-}$satisfies the following properties:

$$
\begin{aligned}
& T \in \mathcal{U}_{-}(X, Y) \text { and } S \in \mathcal{U}_{-}(Y, Z) \Rightarrow S T \in \mathcal{U}_{-}(X, Z) . \\
& T \in \mathcal{U}_{-}(X, Y) \text { and } K \in \mathcal{U}^{d}(X, Y) \Rightarrow T+K \in \mathcal{U}_{-}(X, Y) \\
& T \in \mathcal{U}_{-}(X, Y) \Rightarrow Y / \overline{R(T)} \text { contains no complemented copies of } \ell_{1} .
\end{aligned}
$$

(c) An operator $T \in \mathcal{B}(X, Y)$ with closed range and cokernel $Y / R(T)$ containing no complemented copies of $\ell_{1}$ belongs to $\mathcal{U}_{-}$. This is consequence of Corollary 2.5 and duality.

(d) We have that $T^{*} \in \mathcal{U}_{-}$implies $T \in \mathcal{U}_{+}$, because $T$ is a restriction of $T^{* *}$. However, the converse implication is not true. If we consider a Banach space $Z$ containing no copies of $c_{0}$ such that $Z^{* *}$ contains a copy of $c_{0}$, then the zero operator $0_{Z}$ in $Z$ belongs to $\mathcal{U}_{+}$, but its second conjugate $0_{Z}^{* *}$ does not.

We can take as $Z$ the hereditarily reflexive predual of $\ell_{1}$, obtained by Bourgain and Delbaen [3].

Now we give a perturbative characterisation for $\mathcal{U}_{-}$.

THEOREM 2.19. An operator $T \in \mathcal{B}(X, Y)$ belongs to $\mathcal{U}_{-}$if and only if for every compact operator $K \in \mathcal{B}(X, Y)$, the cokernel $Y / \overline{R(T+K)}$ contains no complemented copies of $\ell_{1}$.

PROOF: The direct implication follows from Remark 2.18. For the converse, assume $T \notin \mathcal{U}_{-}$; equivalently, $T^{*} \notin \mathcal{U}_{+}$. By Proposition 2.7 , we can select a sequence $\left(f_{n}\right)$ in $Y^{*}$ equivalent to the unit vector basis of $c_{0}$ and such that $\lim _{n \rightarrow \infty}\left\|T^{*} f_{n}\right\|=0$.

Using a result of Johnson and Rosenthal [11, Remark 3.1] we can select a subsequence $\left(g_{n}\right)$ of $\left(f_{n}\right)$ and a bounded sequence $\left(y_{n}\right)$ in $Y$ such that $g_{k}\left(y_{l}\right)=\delta_{k l}$. Moreover, we can assume that $\left\|T^{*} g_{n}\right\|\left\|y_{n}\right\|<2^{-n}$. Therefore, the expression

$$
K x:=\sum_{n=1}^{\infty}\left(T^{*} g_{n}\right)(x) y_{n}
$$

defines a compact operator $K \in \mathcal{B}(X, Y)$ whose conjugate is given by

$$
K^{*} f=\sum_{n=1}^{\infty} f\left(y_{n}\right) T^{*} g_{n}
$$

Thus $\left(g_{n}\right)$ is contained in $N\left(T^{*}+K^{*}\right)=[Y / \overline{R(T+K)}]^{*} ;$ hence $Y / \overline{R(T+K)}$ contains a complemented copy of $\ell_{1}$.

Now we can give algebraic characterisations of $\mathcal{U}_{-}$. Note that the identity $I_{X}$ of a Banach space $X$ belongs to $\mathcal{U}^{d}$ if and only if $X$ contains no complemented copies of $\ell_{1}$.

Corollary 2.20. For $T \in \mathcal{B}(X, Y)$, the following assertions are equivalent:

$$
\text { (a) } T \in \mathcal{U}_{-} \text {; }
$$


(b) for every $Z$ and every $A \in \mathcal{B}(Y, Z)$, if $A T \in \mathcal{U}^{d}$ then $A \in \mathcal{U}^{d}$;

(c) for every closed subspace $M$ of $Y$, if $q_{M} T \in \mathcal{U}^{d}$, then $Y / M$ contains no complemented copies of $\ell_{1}$.

Proof: (a) $\Rightarrow(b)$ If $T \in \mathcal{U}_{-}$and $A T \in \mathcal{U}^{d}$ then $T^{*} \in \mathcal{U}_{+}$and $T^{*} A^{*} \in \mathcal{U}$. Theorem 2.9 gives that $A^{*} \in \mathcal{U}$, hence $A \in \mathcal{A}^{d}$.

(b) $\Rightarrow$ (c) Assume $M$ is a subspace of $Y$ such that $q_{M} T \in \mathcal{U}^{d}$. Hypothesis (b) leads to $q_{M} \in \mathcal{U}^{d}$; equivalently, the dual of $Y / M$ contains no copies of $c_{0}$.

(c) $\Rightarrow$ (a) Assume $T \notin \mathcal{U}_{-}$. By Theorem 2.19, there is a compact operator $K \in$ $\mathcal{B}(X, Y)$ such that $[Y / \overline{R(T+K)}]^{*}$ contains a copy of $c_{0}$. Let $M:=\overline{R(T+K)}$. Since $K$ is compact, $-q_{M} K=q_{M} T$ is compact, so $q_{M} T \in \mathcal{U}^{d}$, but $q_{M} \notin \mathcal{U}^{d}$.

An operator $T \in \mathcal{B}(X, Y)$ is said to be lower semi-Fredholm, denoted $T \in \mathcal{F}_{-}[13]$ or cotauberian [19], denoted $T \in \mathcal{T}_{-}$, if $T^{*}$ belongs to $\mathcal{F}_{+}$or $\mathcal{T}_{+}$, respectively. Both classes are semigroups and admit perturbative characterisations.

Proposition 2.21. [10, Theorem 1.b] Given $T \in \mathcal{B}(X, Y)$, we have that $T \in \mathcal{F}_{-}$(respectively, $\mathcal{T}_{-}$) if and only if for every compact operator $K \in \mathcal{B}(X, Y)$ the cokernel $Y / \overline{R(T+K)}$ is finite dimensional (respectively, reflexive).

Corollary 2.22. For every pair of Banach spaces $X, Y$ we have

$$
\mathcal{F}_{-}(X, Y) \subset \mathcal{T}_{-}(X, Y) \subset \mathcal{U}_{-}(X, Y)
$$

Using the perturbative characterisations for the semigroups $\mathcal{F}_{-}, \mathcal{T}_{-}$and $\mathcal{U}_{-}$we can derive characterisations for some classes of Banach spaces. The proof is analogous to that of Proposition 2.13.

Proposition 2.23. Let $X$ be a Banach space.

(a) Quotients of $X$ containing no complemented copies of $\ell_{1}$ are finite dimensional if and only if for every $Y$ we have $\mathcal{U}_{-}(Y, X)=\mathcal{F}_{-}(Y, X)$.

(b) Quotients of $X$ containing no complemented copies of $\ell_{1}$ are reflexive if and only if for every $Y$ we have $\mathcal{U}_{-}(Y, X)=\mathcal{T}_{-}(Y, X)$.

(c) Reflexive quotients of $X$ are finite dimensional if and only if for every $Y$ we have $\mathcal{T}_{-}(Y, X)=\mathcal{F}_{-}(Y, X)$.

Now we study the perturbation class of $\mathcal{U}_{-}$. Recall that an operator $T \in \mathcal{B}(X, Y)$ is said to be strictly cosingular if a closed subspace $N$ of $Y$ is finite codimensional whenever $R(T)+N=Y$. The perturbation class for the lower semi-Fredholm operators contains the strictly cosingular operators, and it is an open problem whether they coincide [17].

We have observed in Remark 2.18 that the perturbation class for $\mathcal{U}_{\text {- contains }} \mathcal{U}^{d}$. Next we show that these classes coincide for operators acting in the same space.

PROPOSITION 2.24. The perturbation class of $\mathcal{U}_{-}$coincides with $\mathcal{U}^{d}$. 
Proof: Assume that $\mathcal{U}_{-}(X, Y)$ is not empty, and that $A \in \mathcal{B}(X, Y)$ does not belong to $\mathcal{U}^{d}$. It follows from Proposition 2.17 that there exists a subspace $M$ of $X$ isomorphic to $\ell_{1}$ such that the restriction $\left.A\right|_{M}$ is an isomorphism and $A(M)$ is complemented. As in the proof of Proposition 2.14, we get that $M$ is also complemented in $X$, and using the argument in the proof of Proposition 2.15, we may assume that

$$
X=U \oplus M \text { and } Y=V \oplus A(M)
$$

with $U$ and $V$ isomorphic to $X$ and $Y$, respectively.

Now we can define an operator $T \in \mathcal{B}(X, Y)$ such that $\left.T\right|_{M}=\left.A\right|_{M}, T(U) \subset V$ and $\left.T\right|_{U}$ belongs to $\mathcal{U}_{-}$. Clearly $T \in \mathcal{U}_{-}$. However, $T+A \notin \mathcal{U}_{-}$.

\section{Semiembeddings and SEmigroups}

Here we show the relation between operators in $\mathcal{U}_{+}$and semiembeddings. Recall that $T \in \mathcal{B}(X, Y)$ is said to be a semiembedding if $T$ is injective and $T B_{X}$ is closed. This concept, introduced in [16], has found applications in the study of the Radon-Nikodym property [4].

Semiembeddings are not stable under isomorphic renorming of the initial space [16], but it has been proved by Saint-Raymond (see [4, Proposition 1.6]) that an operator $T \in \mathcal{B}(X, Y)$ is a semi-embedding under some equivalent norm for $X$ if and only if it is injective and its range $T(X)$ is an $F_{\sigma}$-set; that is, a countable union of closed sets. These operators are called $F_{\sigma}$-embeddings.

We shall present two examples of semiembeddings not belonging to $\mathcal{U}_{+}$, but we also show that $F_{\sigma}$-embeddings of $X$ belong to $\mathcal{U}_{+}(X, Y)$ if (and only if) $X$ contains no copies of $\ell_{\infty}$.

ExAMPLES. (a) The operator $S \in \mathcal{B}\left(\ell_{\infty}, \ell_{2}\right)$, given by $S\left(x_{n}\right):=\left(x_{n} / n\right)$, is an injective conjugate operator; hence it is a semiembedding. However, $S \notin \mathcal{U}_{+}$because it carries the unit vector basis of $c_{0} \subset \ell_{\infty}$ into a norm null sequence.

(b) For $1 \leqslant p<\infty$, the natural inclusion $i \in \mathcal{B}\left(L_{\infty}[0,1], L_{p}[0,1]\right)$ is a semiembedding; in fact, a sequence in the unit ball of $L_{\infty}$ converging in the $L_{p}$-norm has a subsequence converging almost everywhere to a measurable function which belongs to the unit ball of $L_{\infty}$ too.

However, $i$ is not $\mathcal{U}_{+}$since given a sequence of pairwise disjoint, measurable subsets $C_{n} \subset[0,1]$ with $\mu\left(C_{n}\right)>0$, the corresponding sequence of characteristic functions $\chi_{C_{n}}$ is equivalent in $L_{\infty}$ to the unit vector basis of $c_{0}$, but $\lim _{n \rightarrow \infty}\left\|\chi_{C_{n}}\right\|_{p}=\lim _{n \rightarrow \infty} \mu\left(C_{n}\right)^{1 / p}=0$.

THEOREM 3.1. A Banach space $X$ contains no copies of $\ell_{\infty}$ if and only if for every equivalent norm $|\cdot|$ in $X$, the semiembeddings of $(X,|\cdot|)$ into any Banach space belong to $\mathcal{U}_{+}$. 
Proof: Suppose $T \in \mathcal{B}(X, Y)$ is a semiembedding and $T \notin \mathcal{U}_{+}(X, Y)$. By Proposition 2.7 there exists a sequence $\left(x_{n}\right) \subset X$ equivalent to the unit vector basis of $c_{0}$ and such that $\sum_{n=1}^{\infty}\left\|T x_{n}\right\|<\infty$.

We select a constant $M$ such that for every finite sequence of scalars $\left\{t_{1}, \ldots, t_{n}\right\}$ with $\max _{1 \leqslant i \leqslant n}\left|t_{i}\right| \leqslant M$ we have $t_{1} x_{1}+\cdots+t_{n} x_{n} \in B_{X}$. Since $T\left(B_{X}\right)$ is closed, for every $\left(t_{i}\right) \in \ell_{\infty}$ with $\left\|\left(t_{i}\right)\right\|_{\infty} \leqslant M$ we have that $\sum_{i=1}^{\infty} t_{i} T x_{i}$ is absolutely convergent to some vector $y \in T B_{X}$. Then

$$
\left(t_{n}\right) \in \ell_{\infty} \longrightarrow T^{-1}\left(\sum_{n=1}^{\infty} t_{n} T x_{n}\right) \in X
$$

defines an operator $R \in \mathcal{B}\left(\ell_{\infty}, X\right)$ such that $\|R\| \leqslant M^{-1}$ and $\left.R\right|_{c_{0}}$ is an isomorphism. By a result of Rosenthal (see [14, Proposition 2.f.4]), there exists an infinite subset $A \subset \mathbb{N}$ so that $\left.R\right|_{\ell_{\infty}(A)}$ is an isomorphism. Hence $X$ contains a copy of $\ell_{\infty}$.

Conversely, if $X$ contains a copy of $\ell_{\infty}$, then $X$ is isomorphic to $\ell_{\infty} \times Y$ for some $Y$. Now, if we endow the products $\ell_{\infty} \times Y$ and $\ell_{2} \times Y$ with the supremum norms, we have that $T\left(\left(x_{n}\right), y\right):=\left(\left(x_{n} / n\right), y\right)$ defines a semiembedding of $\ell_{\infty} \times Y$ into $\ell_{2} \times Y$ which is not in $\mathcal{U}_{+}$.

Corollary 3.2. Assume that $X$ contains no copies of $\ell_{\infty}$.

(a) Every semiembedding $T: X \rightarrow Y$ belongs to $\mathcal{U}_{+}$.

(b) If there exists a semiembedding $T: X \rightarrow Y$ and every subspace of $Y$ isomorphic to $c_{0}$ is complemented, then the same is true for $X$.

Next we show that operators of $\mathcal{U}_{+}$defined on $C[0,1]$ or $L_{\infty}[0,1]$ preserve a copy of the whole space.

Proposition 3.3. Suppose $X$ is $C[0,1]$ or $L_{\infty}[0,1]$. Then for every $T \in$ $\mathcal{U}_{+}(X, Y)$ there exists a subspace $M$ of $X$ isomorphic to $X$, such that the restriction $\left.T\right|_{M}$ is an isomorphism.

Proof: Assume $I_{n}$ is a disjoint sequence of closed, non-empty subintervals of $[0,1]$. We denote by $C_{n}$ the subspace of functions with (essential) support contained in $I_{n}$.

If $\left.T\right|_{C_{n}}$ is an isomorphism for some $n$, then have finished. Otherwise we can select a sequence of normalised functions $f_{n} \in C_{n}$, with $\lim _{n}\left\|T f_{n}\right\|=0$. Since $\left(f_{n}\right)$ is equivalent to the unit vector basis of $c_{0}$, we obtain $T \notin \mathcal{U}_{+}$, a contradiction.

Finally we state some open questions.

Problems. Suppose $X$ is $C[0,1]$ or $L_{\infty}[0,1]$, and $Y$ is any Banach space.

(a) Is it true that $T \in \mathcal{U}_{+}(X, Y)$ if there is no normalised disjoint sequence $\left(f_{n}\right)$ in $X$ such that $\lim _{n \rightarrow \infty}\left\|T f_{n}\right\|=0$ ?

(b) Is it true that $\mathcal{U}_{+}(X, Y)$ is open in $\mathcal{B}(X, Y)$ ? 
We refer to [8] for a positive answer to similar questions for tauberian operators on $L_{1}[0,1]$.

\section{REFERENCES}

[1] T. Alvarez and M. González, 'Some examples of tauberian operators', Proc. Amer. Math. Soc. 111 (1991), 1023-1027.

[2] C. Bessaga and A. Pelczyński, 'On basis and unconditional convergence of series in Banach spaces', Studia Math. 17 (1958), 151-164.

[3] J. Bourgain and F. Delbaen, 'A class of special $\mathcal{L}_{\infty}$-spaces', Acta Math. 145 (1980), $155-176$.

[4] J. Bourgain and H.P. Rosenthal, 'Applications of the theory of semi-embeddings to Banach space theory', J. Funct. Anal. 52 (1983), 149-188.

[5] W.J. Davis, T. Figiel, W.B. Johnson and A. Pelczyński, 'Factoring weakly compact operators', J. Funct. Anal. 17 (1974), 311-327.

[6] R. Deville, G. Godefroy and V. Zizler, Smoothness and renormings in Banach spaces (Longman Scientific and Technical, New York, NY, 1993).

[7] M. González, 'Properties and applications of tauberian operators', Extracta Math. 5 (1990), 91-107.

[8] M. González and A. Martínez-Abejón, 'Tauberian operators on $L_{1}(\mu)$ spaces', Studia Math. 125 (1997), 289-303.

[9] M. González and V.M. Onieva, 'Lifting results for sequences in Banach spaces', Math. Proc. Cambridge Phil. Soc. 105 (1989), 117-121.

[10] M. González and V.M. Onieva, 'Characterizations of tauberian operators and other semigroups of operators', Proc. Amer. Math. Soc. 108 (1990), 399-405.

[11] W.B. Johnson and H.P. Rosenthal, 'On $w^{*}$-basic sequences and their application to the study of Banach spaces', Studia Math. 43 (1972), 77-99.

[12] N. Kalton and A. Wilansky, 'Tauberian operators on Banach spaces', Proc. Amer. Math. Soc. 57 (1976), 251-255.

[13] A. Lebow and M. Schechter, 'Semigroups of operators and measures of noncompactness', J. Funct. Anal. 7 (1971), 1-26.

[14] J. Lindenstrauss and L. Tzafriri, Classical Banach spaces I. Sequence spaces (Springer-Verlag, Berlin, Heidelberg, New York, 1977).

[15] R.H. Lohman, 'A note on Banach spaces containing $\ell_{1}$ ', Canad. Math. Bull. 19 (1976), 365-367.

[16] H.P. Lotz, N.T. Peck, H. Porta, 'Semi-embeddings of Banach spaces', Proc. Edinburgh Math. Soc. 22 (1979), 233-240.

[17] A. Pietsch, Operator ideals (North-Holland, 1980).

[18] D. Przeworska-Rolewicz and S. Rolewicz, Equations in linear spaces (P.W.N., Warszawa, 1968).

[19] D.G. Tacon, 'Generalized Fredholm transformations', J. Austral Math. Soc. 37 (1984), 89-97.

Departamento de Matemáticas

Facultad de Ciencias

Universidad de Cantabria

39071 Santander, Spain
Departamento de Matemáticas

Facultead de Ciencias

Universidad de Oviedo

33007 Oviedo, Spain 